\title{
Penicillin G-induced Hemorrhagic Cystitis with Hydronephrosis
}

\author{
Yuichiro Toma ${ }^{1}$, Tetsuya Ishiki ${ }^{1}$, Kazufumi Nagahama ${ }^{1}$, Koichiro Okumura ${ }^{1}$, \\ Tomomasa Kamiyama ${ }^{1}$, Kentaro Kohagura ${ }^{1}$, Ayano Kakihana ${ }^{2}$, Takeshi Tana ${ }^{2}$, \\ Yusuke Ohya ${ }^{1}$ and Shuichi Takishita ${ }^{1}$
}

\begin{abstract}
Irritable urological symptoms with gross hematuria and bilateral lumbar pain developed when the patient received penicillin $G$ for endocarditis. These symptoms were followed by renal insufficiency. A contrastenhanced abdominal computed tomography (CT) scan revealed a thickened bladder wall, bilateral hydroureter and hydronephrosis, suggesting hemorrhagic cystitis complicated with urinary tract obstruction. Urine culture was negative. After discontinuation of penicillin $\mathrm{G}$, all symptoms subsided and renal function recovered; hence, penicillin $\mathrm{G}$ seems to have been associated with hemorrhagic cystitis and acute kidney injury. Positive findings in the drug lymphocyte stimulation test (DLST) for penicillin G were consistent with this diagnosis.
\end{abstract}

Key words: penicillin G, hemorrhagic cystitis, interstitial nehritis, infectious endocarditis

(Inter Med 48: 1667-1669, 2009)

(DOI: 10.2169/internalmedicine.48.1842)

\section{Introduction}

Interstitial nephritis is a well-known complication of penicillin $\mathrm{G}$ and related drugs $(1,2)$. Hemorrhagic cystitis is a rare complication of penicillin $\mathrm{G}$, but occasionally causes kidney injury. Here, we report a patient who developed hemorrhagic cystitis with urinary tract obstruction during treatment for endocarditis with penicillin $\mathrm{G}$.

\section{Case Report}

A 49-year-old man visited the clinic because of a 2-week fever with shortness of breath and orthopnea. The findings of physical examination and chest X-ray suggested congestive heart failure. A pansystolic murmur was heard, most intensely at the apex. Echocardiography revealed severe mitral valve regurgitation. He was referred to our hospital and admitted. His temperature was 38.7 degrees Celsius, blood pressure $126 / 70 \mathrm{mmHg}$, pulse rate 116 per minute. The pansystolic pericardial murmur was classified as grade 4 . The sudden development of mitral valve regurgitation and two- week fever of unknown origin were strongly suggestive of endocarditis, although two sets of blood cultures were negative and we could not detect vegetation. We began to treat the patient with penicillin $\mathrm{G}$ ( 4 million units every 4 hours), gentamicin (180 mg every 8 hours) and diuretics (Fig. 1). His fever declined soon after starting the antibiotics, followed by prompt resolution of congestive heart failure. We continued only penicillin $G$ at the same dose from the 14th day of hospitalization. Dysuria and frequent urination developed from the 16th day, followed by gross hematuria a few days later. Urinalysis, which had been normal previously, showed urine protein $3+$ and numerous polymorphonuclear leukocytes. Although no bacteria were found in urine culture or a Gram stain of the urine sediment, we began tosufloxacin $400 \mathrm{mg}$ per day from the 18th hospital day, based on a presumptive diagnosis of bacterial prostatitis. However, bilateral lumbago developed starting the 20th day of hospitalization. We gave diclofenac $150 \mathrm{mg}$ per day for the pain. Contrast-enhanced abdominal CT revealed mild bilateral hydronephrosis, hydroureter, and thickening of the ureteral wall and bladder wall (Fig. 2). The thickened bladder wall with irritable urological symptoms was suggestive of cysti-

${ }^{1}$ The Third Department of Internal Medicine, University of the Ryukyus, Okinawa and ${ }^{2}$ Shuri Jokamachi Medical Clinic, Naha Received for publication November 5, 2008; Accepted for publication May 28, 2009

Correspondence to Dr. Yuichiro Toma, asikaga@yahoo.co.jp 


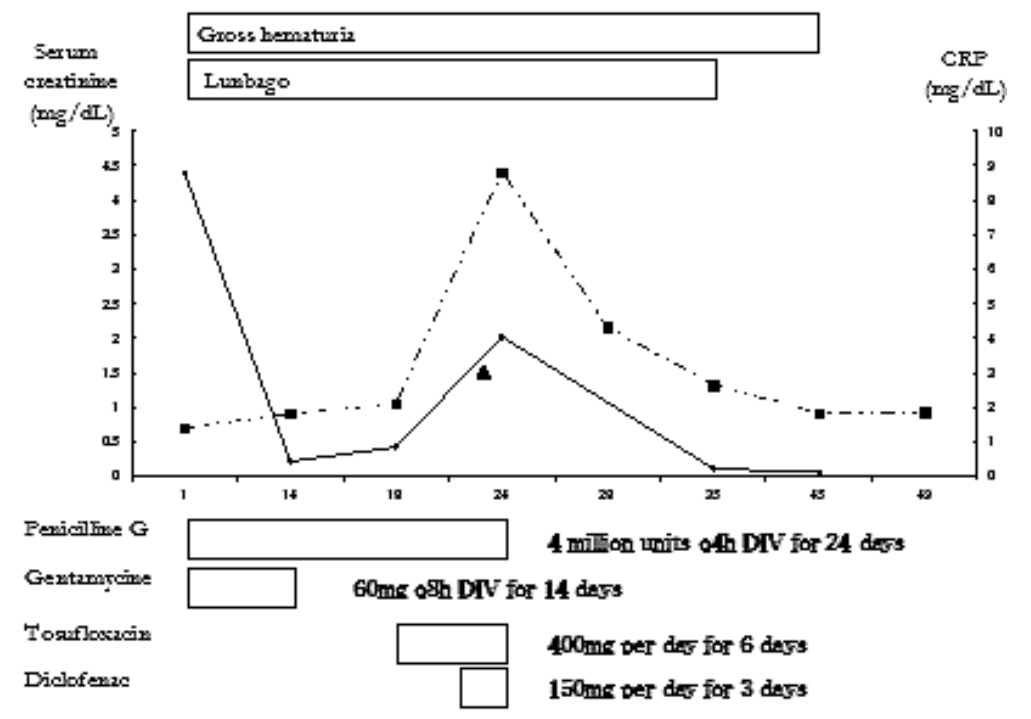

Figure 1. Clinical time course. Upper panel shows the changes in the symptoms, serum creatinine (full line) and CRP (dotted line). Lower panel shows the medication. Arrow indicates the time of the contrast-enhanced abdominal CT.

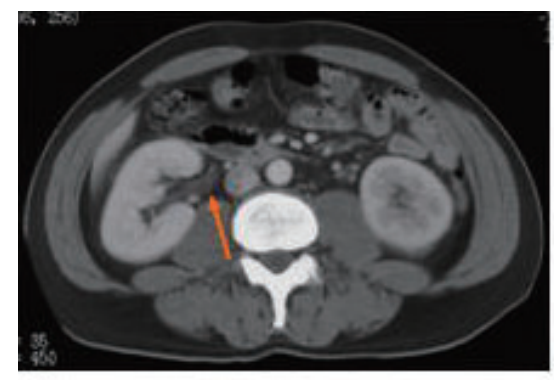

A

$C$

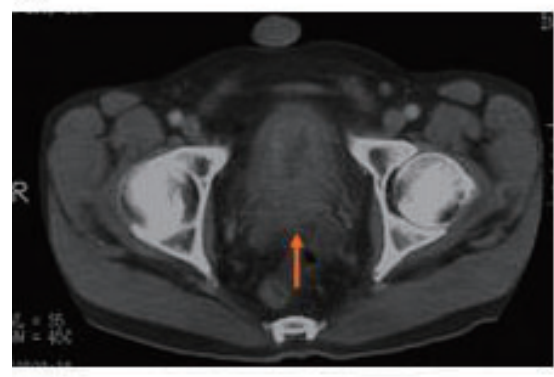

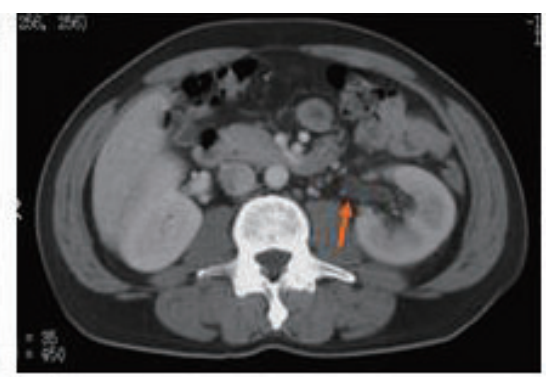

B

D

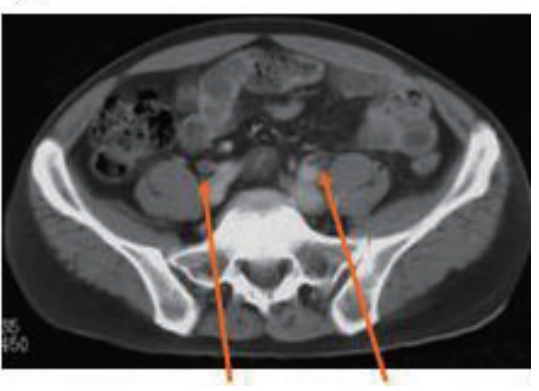

Figure 2. Contrast-enhanced abdominal computed tomography on the 21st of hospitalization day. Bilateral hydronephrosis (A, B arrows), thickened bladder wall (C, arrows) and thickened ureteral walls $(\mathrm{D}$, arrow) were observed.

tis. Under the tentative diagnosis of urinary tract infection developing from prostatitis, we continued treatment with antibiotics including penicillin G. On the 24th hospitalization day, serum creatinine had increased from $0.7 \mathrm{mg} / \mathrm{dL}$ at the admission to $4.4 \mathrm{mg} / \mathrm{dL}$. Urine $\beta-2$ microglobulin (MG) and urine N-acetyl- $\beta$-D-glucosaminidase (NAG) had become elevated to $12.8 \mathrm{mg} / \mathrm{L}$ and $17.5 \mathrm{IU} / \mathrm{L}$, respectively. Moreover, we found newly developed eosinophilia $(781 / \mu \mathrm{L})$, and the drug lymphocyte stimulation test (DLST) for penicillin $G$ was positive. Based on these findings, it was suggested that penicillin $\mathrm{G}$ might be involved in acute kidney injury (AKI). We discontinued diclofenac and penicillin $\mathrm{G}$ and changed to linezolid and aztreonam, respectively. After discontinuation of the former drugs, gross hematuria and lumbago were improved and serum creatinine decreased, reaching $0.91 \mathrm{mg} / \mathrm{dL}$ on the 45th day. We also found that eosinophilia, urine white blood cells, urine red blood cells, urine $\beta-2 \mathrm{MG}$ and urine NAG were improved to within the normal range. We confirmed that hydronephrosis and hydroureter were improved on abdominal CT. After the patient received mitral valve plastic surgery, he was discharged. About 1 year after discharge from our hospital the patient was well with normal urine sediment, no eosinophilia and stable renal function. 


\section{Comments}

In this case, AKI seemed to be due to ureteral obstruction. Tubulo-interstitial nephritis (TIN) is a well-known complication of penicillin as a cause of AKI (3). On the other hand, hemorrhagic cystitis is a quite rare complication of penicillin. We could find only two case reports which showed that penicillin could induce hemorrhagic cystitis (4, 5). One of the two cases was reported to be complicated by AKI as in the present case (5). Although the precise mechanism of toxicity in penicillin-induced hemorrhagic cystitis is not known, it is possible that penicillin or its metabolites may directly irritate the bladder and ureter or that immunoglobulin, which has a cross-reaction to both penicillin and those tissues, might attack them (6). In the present case, the immunological reaction to penicillin $\mathrm{G}$ was likely involved in the mechanism, based on the positive finding of DLST for penicillin $\mathrm{G}$ and the increased concentration of eosinophils in blood. Severe non-bacterial hemorrhagic cystitis is known to be induced by viral infection such as adenovirus, especially in children, and also by some drugs such as cyclophosphamide. Viral cystitis might be one of the most important differential diagnoses. But adenovirus causes hemorrhagic cystitis primarily among children, and other viruses such as BK virus are usually observed in severely immunosuppressed patients. Stem cell treatments and solid organ transplantation can create such conditions (7). The reported patient was in critical condition, but it was a different condition from that in reported viral cystitis. As mentioned above, penicillin G-induced hemorrhagic cystitis is quite rare; therefore, we did not consider it as a cause of the symptoms. The resultant delayed diagnosis might have been associated with the AKI in this case. We should have changed penicillin G to an alternative antibiotic soon after the appearance of irritable urological symptoms, which were unlikely due to infection. Therefore, it is important to be aware of cystitis as an adverse effect of penicillin if we encounter the development of urological irritable symptoms during penicillin treatment.

\section{References}

1. Colvin RB, Burton JR, Hyslop NE Jr, Spitz L, Lichtenstein NS Letter: Penicillin-associated interstitial nephritis. Ann Intern Med 81: 404-405, 1974.

2. Appel GB, Garvey G, Silva F, Francke E, Neu HC, Weissman J. Acute interstitial nephritis due to amoxicillin therapy. Nephron 27: 313-315, 1981

3. Handa SP. Drug-induced acute interstitial nephritis: report of 10 cases. CMAJ 135: 1278-1281, 1986.

4. Adlam D, Firoozan S, Gribbin B, Banning AP. Haemorrhagic cys- titis and renal dysfunction associated with high dose benzylpenicillin. J Infect 40: 102-103, 2000.

5. Cook FV, Farrar WE Jr, Kreutner A. Hemorrhagic cystitis and ureteritis, and interstitial nephritis associated with administration of penicillin G. J Urol 122: 110-111, 1979.

6. Marx CM, Alpert SE. Ticarcillin-induced cystitis. Cross-reactivity with related penicillins. Am J Dis Child 138: 670-672, 1984.

7. Paduch DA. Viral lower urinary tract infections. Curr Urol Rep 8: 324-335, 2007.

(C) 2009 The Japanese Society of Internal Medicine http://www.naika.or.jp/imindex.html 